\title{
G - CODE BASED DATA RECEIVING AND CONTROL SYSTEM
}

\author{
Amar Baliram Chavan', Vikram Gokuldas Hatkar² \\ ${ }^{1}$ Assistant Professor, Dept. Of Electronics Engineering, MAEER's MITAOE, Alandi(D),Pune, (M.S.), India \\ ${ }^{2}$ Assistant Professor, Dept. of Electronics \& Communication, G. S. Mandal's MIT, Aurangabad, (M.S.), India
}

\begin{abstract}
Today in engineering field technical research among the students are increased. To design and develop such hardware projects we may need to collect real time data from Data Acquisition System. These Systems are manufactured by very few companies in India. These systems are very expensive for the students. Hence students are in need of low cost data acquisition system to perform their research work. In this paper we have explained and implemented a Data Acquisition system (DAQ) using ATmegal6 microcontroller. The GUI and data processing for DAQ system has been designed on LabVIEW and the system has been tested for different tasks.
\end{abstract}

Keywords : Data Acquisition; LabVIEW; G-code; AVR ATmega-16;

\section{INTRODUCTION}

Today the technical education in India is increasing, so the quality research at the student level is rising up. To produce some useful hardware systems we require to acquire the data in real time. The acquisition of data is performed using dedicated system which is called Data Acquisition system. In India there are available Data Acquisition systems are very costly for students. It is requires to make available a cost effective or low cost system which can suite according to their proposed work. In this paper we have designed and developed a prototype of Data Acquisition system (DAS) using AVR. The software is used for data acquisition purpose is LabVIEW and the system has been used for different tasks and under different conditions.

\subsection{Data Acquisition}

The sampling process of signals that detects physical quantities and transforms the digital samples into digital value that can be handled using computer (LabVIEW).

Every Data Acquisition System includes

- Sensors which converts physical signal to electrical signals.

- Signal preparing circuit that converts the signal to easily convert into digital form.

- A to D Converter circuit that converts signal into analog into digital form.

- Data Acquisition applications handled by software programs designed using some programming languages.

\section{PROPOSED SYSTEM}

\subsection{Block Diagram}

The block schematic for this system is as follows:

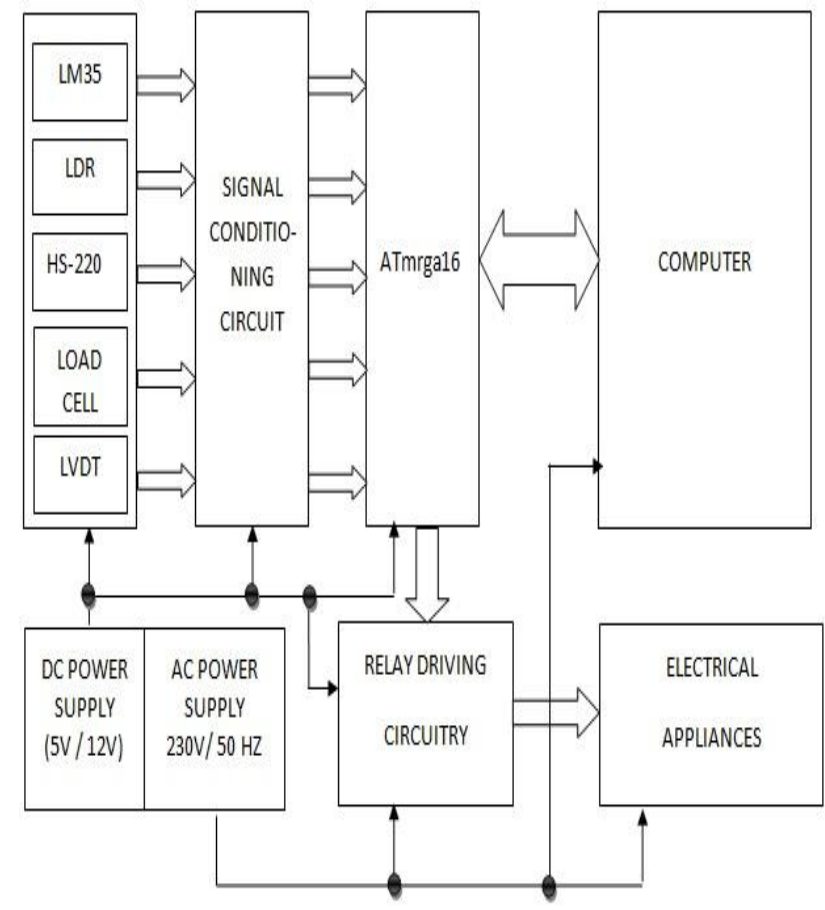

Fig 1: Block Diagram of proposed system

\section{Source:}

Data acquisition is the physical measurend quantity like heat, intensity of light, gas pressure, liquid flow, and force. Without mentioning to the type of physical quantity to be send, the physical form that is to be send must first be converted in such state that can be sampled by a Data acquisition System. The task of performing such conversions is being done by sensors.

A sensor, kind of transducer, which converts a physical quantity into a equivalent electrical signal (e.g., a acquisition system to measure differing properties depends on having correct sensors to detect the various properties to be measured. Signal preparing is required when the signal from the sensor is not meeting the requiremnt of DAQ hardware. 
The signal may required to filter or enhanced in most cases. Also these analog signals are vlunrable to noise can be converted to differential signals. And then these signals can be encoded, hence transmission errorscan be reduced and corrected.

\section{DAQ Software:}

DAQ software is required to govern DAQ hardware interfaced with a PC. In such systems read and write operations on the hardware are done by device drivers. Supervisor application is developed for Data Acquisition and Control purpose. Program is created in engineering environment LabVIEW (Laboratory Virtual Instrument Engineering Workbench). LabVIEW has been chosen because it allows us to create complicated application in short time, programming in graphical language, delivers different controls to representing the data, easy implementing serial communication and obtaining information from the controller. LabVIEW (Laboratory Virtual Instrument Engineering Workbench) is crossplatform a graphic-based programming language developed by National Instruments. The unique NI LabVIEW graphical development environment features interactive assistants, code generation and connectivity to many devices for easily gathering data. Its graphical nature makes it ideal for test and measurement (T\&M), automation, instrument control, data acquisition and data inquiry applications.

\section{DAQ Hardware:}

DAQ hardware that interfaces between the sensor output and computer. It is some kind of module which can be attached with the computer via parallel port or serial port or universal serial bus port. DAQ systems also incorporate the components like ADC, DAC. These are used by a controller that can execute some small codes. A microcontroller is beneficial as compared to hard wired systems; also it is cheaper than a CPU because it is allowed to restrict it with simple polling routines. E.g. holding for ADC/DAC till end, transfer value to RAM, switch the multiplexer, obtain TTL input, let DAC go ahead with voltage ramp.

\section{DESIGN OF SOFTWARE}

Throughout the development of this project we used VMLAB to write, assemble, execute, and debug programs. For testing the Hardware of Data Acquisition system we have used window's Hyper-Terminal program as well as LabVIEW

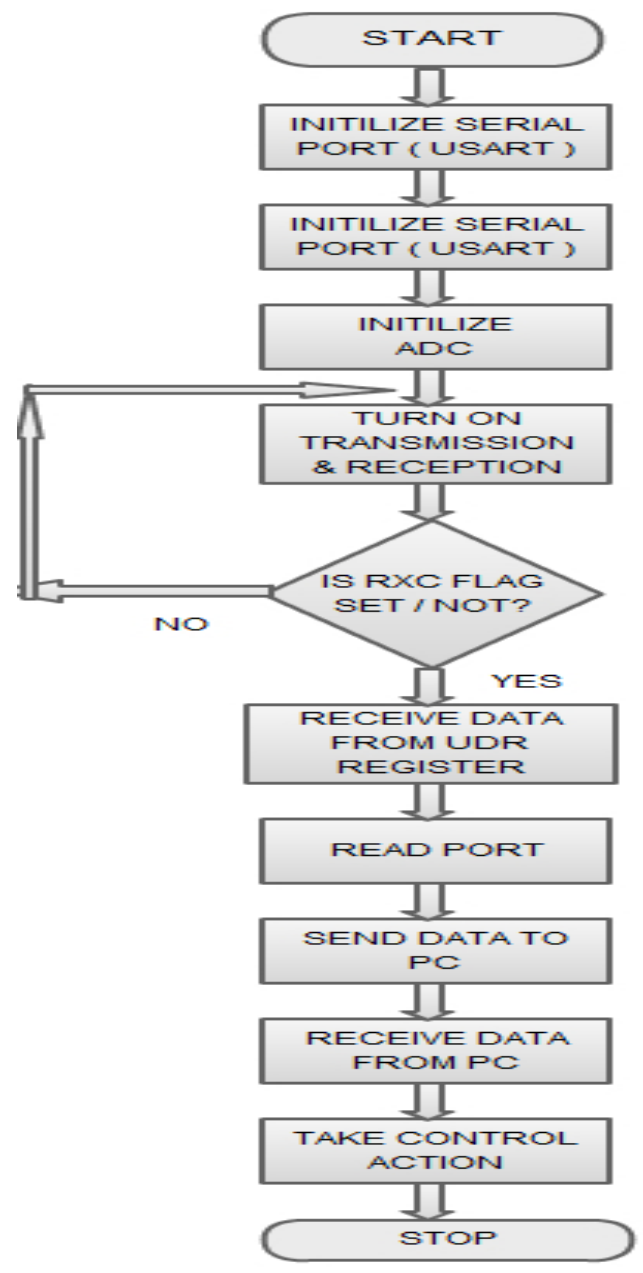

Fig 2: Algorithm of proposed system

VMLAB provides a true virtual microcontrollers design lab, in which the hardware and the software are co-simulated, making unnecessary the in-circuit-emulator.VMLAB has nothing to do with other software simulators, available from microcontrollers makers or other tools vendors. Although some of them talk about I/O simulation, by means of textual scripts, they are far away to perform a full system hw/sw cosimulation, even more, if we talk about combining software simulation with analog simulation.

The only possible comparison to VMLAB, as design tool, is a top class in-circuit emulator + a top class IDE + top class logic analyzer/digital scope, all working together. But even so, VMLAB provides many advantages over this solution 


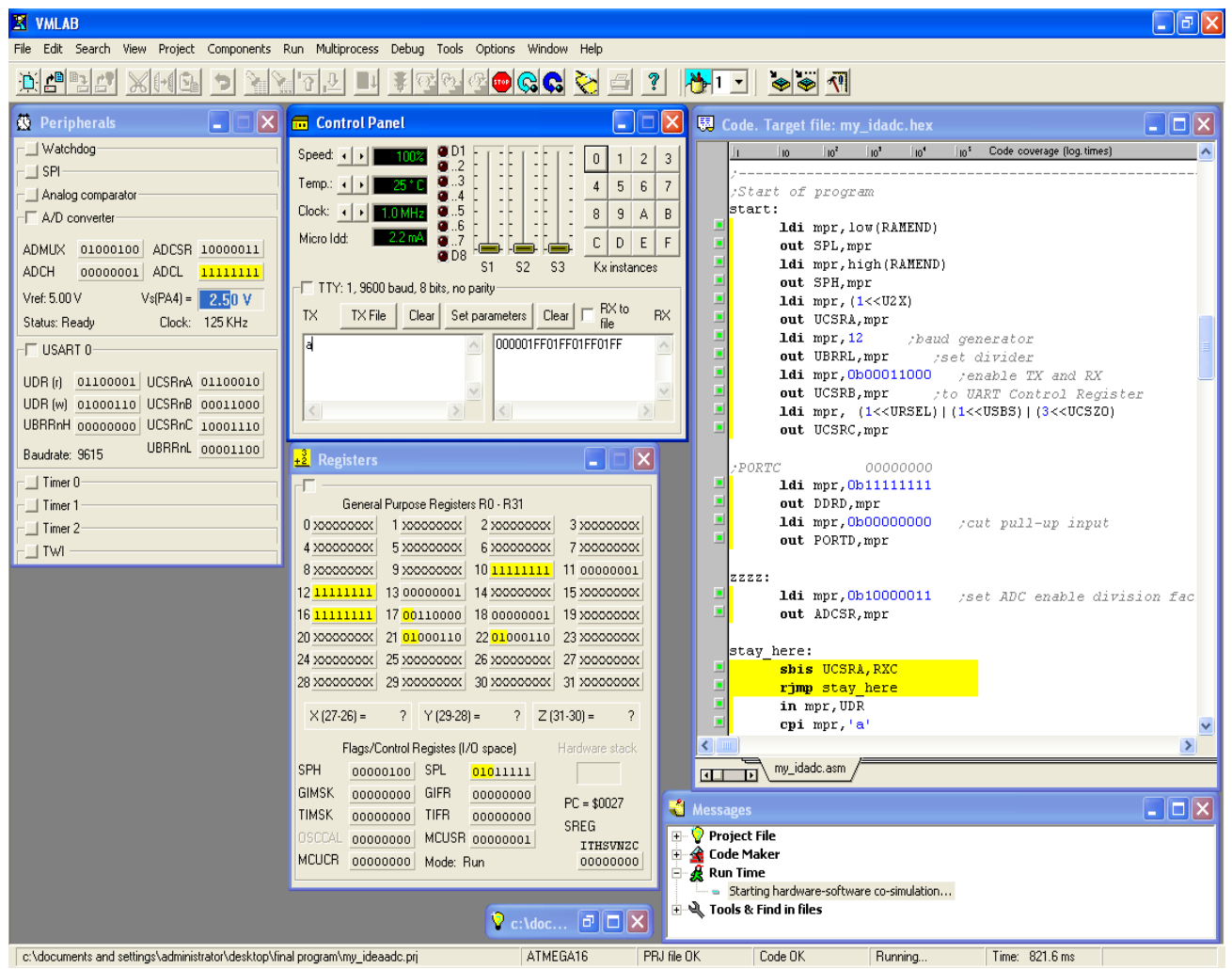

Fig 3: Debugging Window of VMLab

\subsection{LabVIEW (DAQ Software):}

The system is based on LabVIEW Software that holds all the information for all the sensors and their control action. A VI (Virtual Instrument) consists of a Front Panel(Fig. 4), Block Diagram, and an icon that represents the program. The Front Panel is used to display controls-inputs and indicators-outputs for the user, so named because it can simulate the front panel of a physical instrument.

The Block Diagram contains the code for the VI constructed in LabVIEW's graphical programming language in Fig. 5

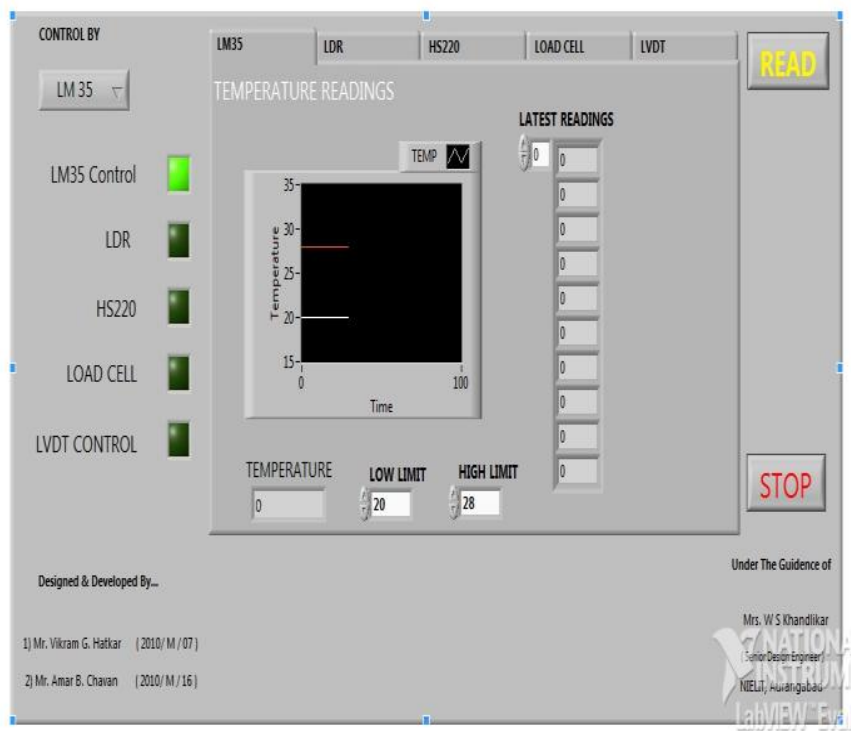

Fig 4: Front Pannel VI (Virtual Instrument) of System
Devices (Indicating Blubs in this system) are controlled according to given set points by user. As obtained data values crosses limits given in the form of set points; the LabVIEW will send a data byte to controller. Then controller senses the data byte from LabVIEW and turns the respective bulb 'on' / 'off' using solid state relays. One can replace the indicating bulbs by any electrical applience which can be operated on $\mathrm{AC}(230 \mathrm{~V} / 50 \mathrm{~Hz})$ or $\mathrm{DC}$ power supply. This results in significant productivity improvements over conventional programming languages. Application which is created in LabVIEW is called VI (Virtual Instrument) because their appearance and operation imitate actual instruments. 


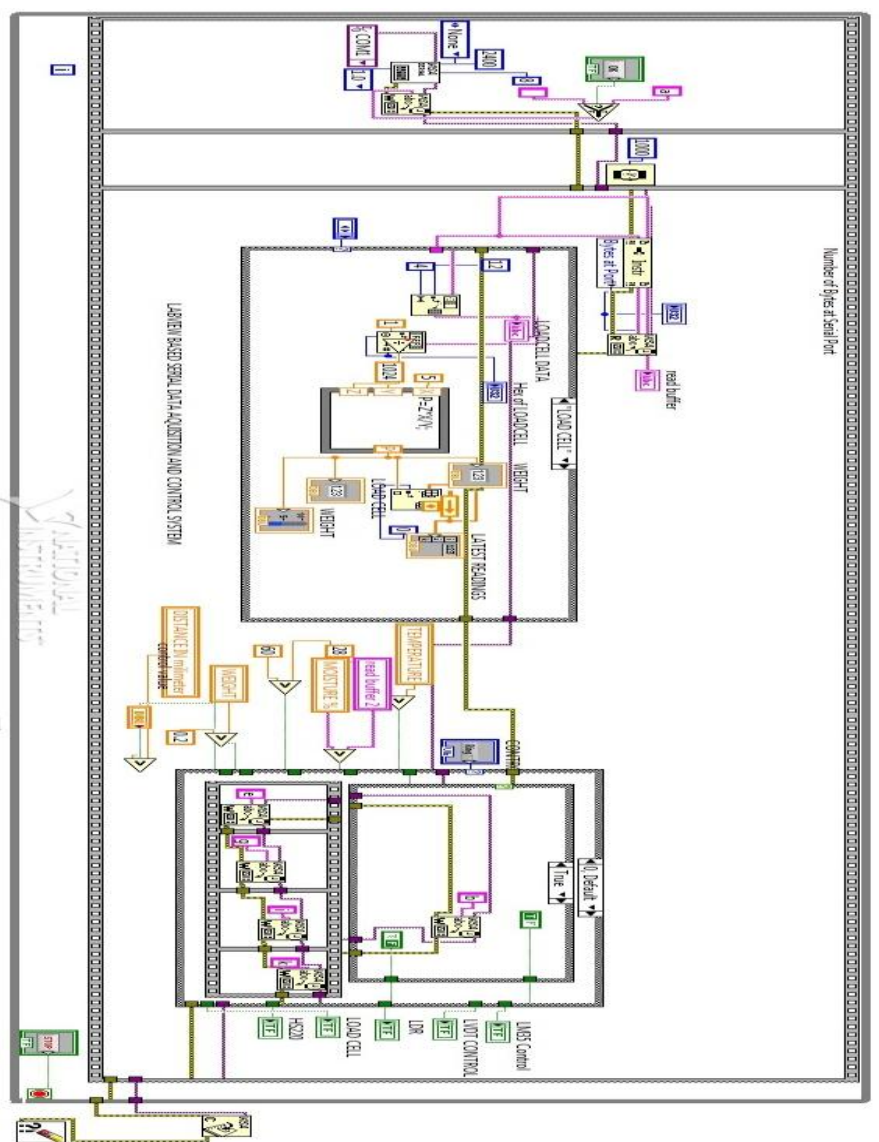

Fig 5: Block Diaram of VI (Virtual Instrument) of System

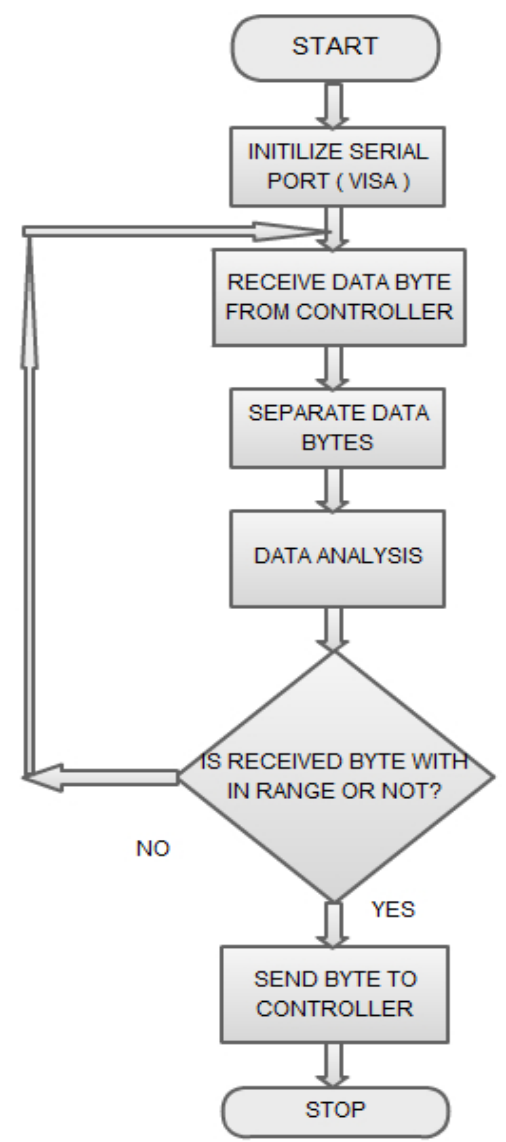

Fig 6: Algorithm of LabVIEW Code.

\section{DESIGN OF HARDWARE}

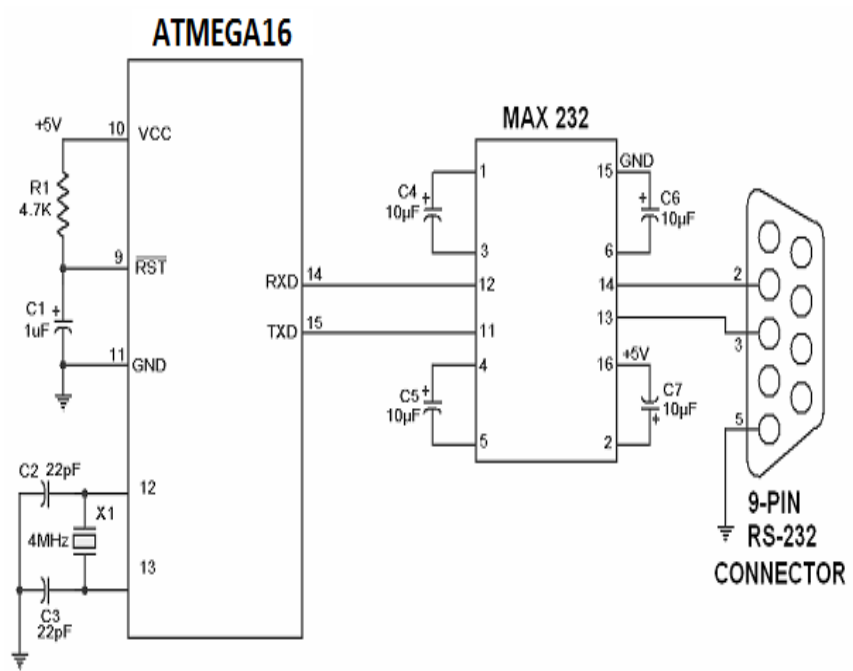

Fig 7: Interfacing of ATmega16 to RS-232(PC).

Microcontroller can interprete only digital languages. However, Almost all the inputs for the microcontroller are analogs by nature, i.e., continuously varies with time. To recognize the input by microcontroller, analog to Digital Converter (ADC) is used. With reference to the name this peripheral hardware collects the analog signals from the sensors and converts it into the controller recognizable form, the microcontroller then handles the information and gives expected throughput at output.

Data Acquisition \& Control System includes control action, hence relay drive unit has been designed. A simple relay control operation is shown in fig below.

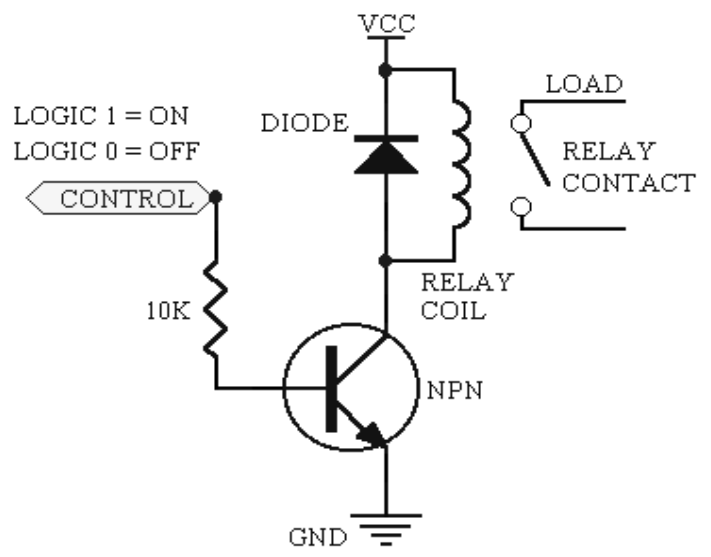

Fig 8: Relay Driving Circuitry.

In LabVIEW, as sensed signal value crosses upper and lower limits (setpoints) provided by user, then LabVIEW sends a corresponding control signal to microcontroller circuitry via serial port. The microcontroller then sends control signal to respective relay to turn 'on' or 'off' perticular indicating bulb. One can connect electrical applience instead of indicating bulbs. These electrical bulbs are controlled by solid state relays which are driven by control signals sent by microcontroller. 
Some snapshots of the system are as follows :

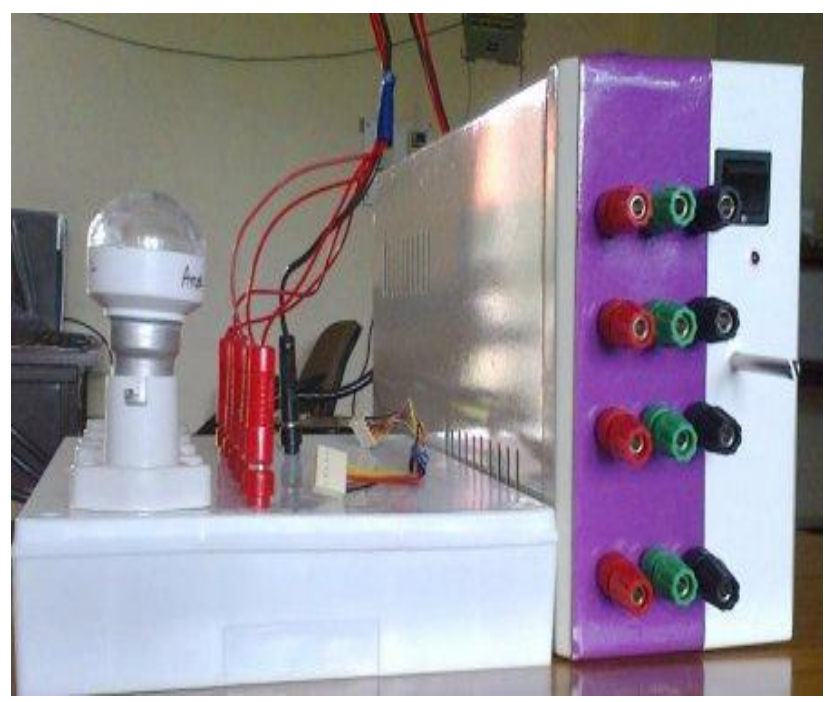

Fig 9: DAQ System with Indicating bulbs.

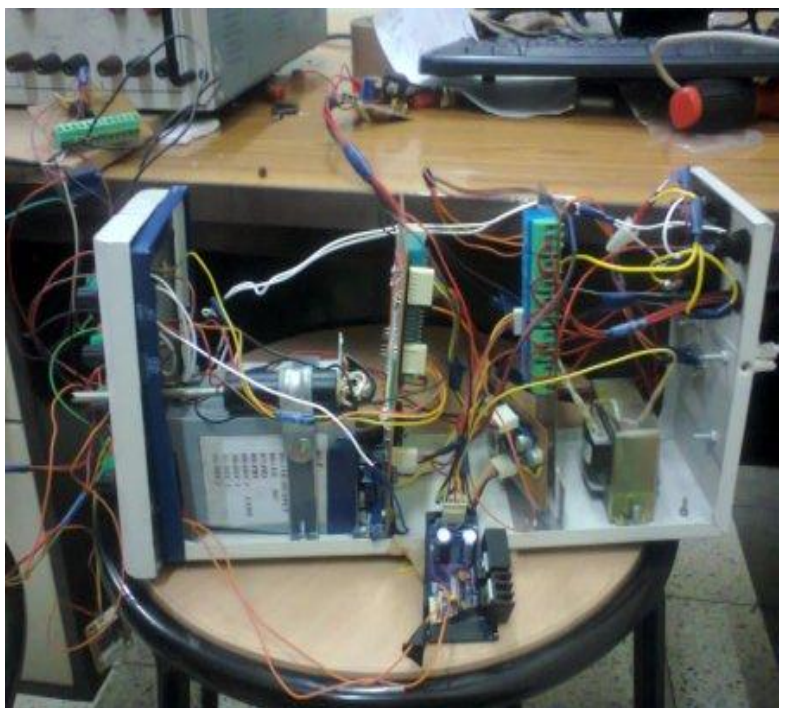

Fig 10: Electronic circuits mounted on pannel.

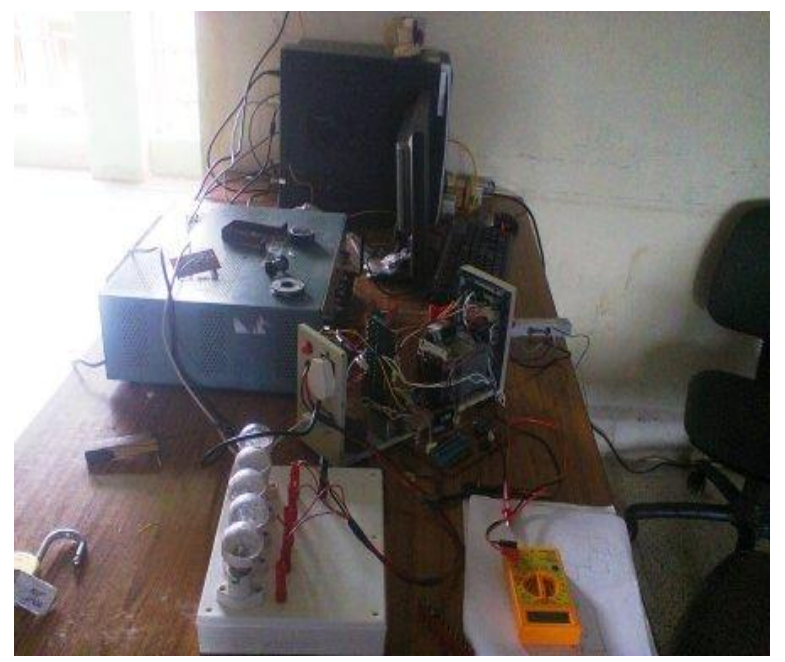

Fig 11: Experimental Setup

\section{CONCLUSION}

The Study carried out in this report demands a Data Acquisition \& Control System to be used as a trainer kit for students learning LabVIEW. Hence we introduced a low cost Data Acquisition \& Control System that obtain sensor signals serially and controls devices according to set points given.

This system is used for some applications where input signal dose not varying rapidly and accuracy is not required.

This concept discussed here is successfully designed developed and tested. This concept can be further utilized for LabVIEW Based projects as future scope of this system.

\section{REFERENCES}

[1] Controlling electrical appliances through an AVR microcontroller based on external sensor stimuli A. DROSOPOULOS1, D.PROTOPAPADAKIS Technical Education Institute (TEI) Patras Department of Electrical Engineering M. Alexandrou 1, 26334 Patras, GREECE

[2] Low Cost Data Acquisition System for Students by Sachin Sharma and Gaurav Kumar ,instrumentation \& Control Engineering National Institute of Technology, Jalandhar

[3] Kalyanramu Vemishetty," Wireless data acquisition system", thesis, submitted in Virginia polytechnic institute and state university

[4] Datasheet of Atmel AVR ATmega8 microcontroller. www.atmel.com/atmel/acrobat/doc2486.pdf.

[5] www.datasheetcatalog.org/datasheet/texasinstruments /max232.pdf, datasheet of MAX 232 IC.

\section{BIOGRAPHIES}

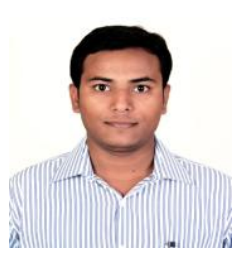

Mr. Amar Chavan is born at Udgir, Maharashtra, India. Presently he is working at MAEER's MITAOE, Alandi(D), Pune. Also he did his B.tech in Electronics and Communication Engineering from Dr. Babasaheb Ambedkar Marathwada University, Aurangabad. His area of interest includes Digital Electronics, Power Electronics, Instrumentation and system designing.

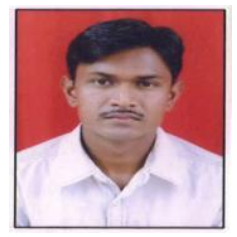

Mr. Vikram Hatkar is presently working at G. S. Mandal's MIT, Aurangabad. Also he did his B.tech in Electronics and Telecommunication Engineering from Dr. Babasaheb Ambedkar Marathwada University, Aurangabad. His area of interest includes Digital Electronics, Embedded Systems. 